\title{
De quoi va-t-on parler ? De la monnaie...
}

\section{Michel Morineau}

\section{OpenEdition}

\section{Journals}

Édition électronique

URL : http://journals.openedition.org/ress/213

DOI : $10.4000 /$ ress.213

ISSN : 1663-4446

\section{Éditeur}

Librairie Droz

\section{Édition imprimée}

Date de publication : 1 juillet 2007

Pagination : 81-98

ISBN : 978-2-600-01155-6

ISSN : 0048-8046

Référence électronique

Michel Morineau, «De quoi va-t-on parler ? De la monnaie... », Revue européenne des sciences sociales [En ligne], XLV-137 | 2007, mis en ligne le 01 juillet 2010, consulté le 04 mai 2019. URL : http:// journals.openedition.org/ress/213; DOI : 10.4000/ress.213 
Michel MORINEAU

\section{DE QUOI VA-T-ON PARLER? DE LA MONNAIE...}

De quoi va-t-on parler? Et non de quoi parle-t-on ? C'est le véritable titre de cette communication. Ainsi conçue, elle avait vocation d'ouverture, d'appel à un échange et à une réflexion collective autour du thème fondamental de la rencontre, présente dans l'arrière-plan plus ou moins rapproché de chacun des exposés. Car, de facto, on en parlerait beaucoup. Elle serait évoquée, invoquée implicitement ou explicitement, par tous les participants. Mais s'inquiéteraient-ils de savoir s'ils étaient foncièrement d'accord entre eux sur l'image qu'ils s'en font et sur l'entité à laquelle ils se référeraient? Si le «personnage historique » que l'on serait invité à rencontrer à tout bout de siècle comme à tout bout de champ avait bien été identique à lui-même, quant au fond, sans altération, imperturbablement? La réponse serait-elle négative que la monnaie resterait semblable, comme elle l'est très souvent dans les ouvrages qui lui sont consacrés, à une princesse lointaine, dissimulée derrière des voiles épais, dont on connaîtrait l'existence, dont on révérerait le pouvoir, dont on célébrerait la beauté, dont on ne désemparerait pas de parler, mais dont on s'abstiendrait d'approcher pour en discerner les traits et s'en faire une idée probe. Ce serait dommage. Pourquoi ne pas saisir l'occasion présente d'en faire le tour et d'en avoir le cœur net, pour une fois et dans la mesure du possible?

Bien entendu, l'entreprise n'est pas entièrement neuve. De longtemps, on a attiré l'attention sur l'ambiguïté du terme en français, qui a tantôt un sens général, tantôt celui d'une unité individualisée, tantôt encore celui d'appoint. Confusion dont on se débarrasse sans trop de peine avec des subterfuges comme la distinction induite par l'alternance du singulier et du pluriel : monnaie et monnaies ; des équivalences taxinomiques : espèces, numéraire ; des adjectifs : la petite et, même, la grosse monnaie. Traquenards, s'il en fut, puérils, auxquels on ne s'arrêtera pas et pas davantage à ces notions plus élaborées que sont la monnaie réelle, la monnaie de compte, la monnaie métallique, la monnaie fiduciaire, la monnaie scripturale, etc. Quitte, néanmoins, à nous en servir dans le cours du développement pour lever le doute qui planerait sur leur qualité : purement formelles ou, éventuellement, essentielles. Je m'appliquerai au contraire à l'étude des caractères que l'on considère traditionnellement comme fondamentaux de la monnaie, en général. C'est-à-dire le rôle $1^{\circ}$ d'étalon de valeur, $2^{\circ}$ d'instrument d'échange, $3^{\circ}$ de réserve de la valeur. Je leur adjoindrai celui, plus récemment attribué, de moteur de la vie économique, de la croissance, notamment par les investissements. 


\section{MESURE DE LA VALEUR}

Parmi tous les genres de monnaies, celles d'or sont tenues pour les parangons de la mesure de la valeur. Dans cette fonction, le métal jaune a pour lui l'inaltérabilité, l'homogénéité, la facilité d'être divisé sans perte de substance. Mais on sait qu'il n'a pas été la seule matière à avoir rempli le rôle d'instrument d'échange, voire à avoir été monétisée et, même, monnayée. Possédant pour certaines comme l'argent, le bronze, le cuivre, fût-ce à un moindre degré que « la sueur du soleil », quelques-unes des qualités requises pour satisfaire à l'emploi. Pour d'autres, comme les fèves de cacao des Aztèques, les cauris en Afrique, les pierres des Mélanésiens, etc., sans ombre de ces mérites, il est facile de s'en débarrasser en les renvoyant à une paléomonnaie, espèce de catégorie-placard qui les mettrait d'entrée hors jeu. Le procédé peut passer pour élégant mais il ampute, également d'emblée la notion de monnaie prise dans son existence concrète, qu'il n'entérine dès lors que dans des conditions très définies. En faisant fi, d'une part, du consensus qui a entouré ces parias de l'histoire monétaire ; d'autre part, du sceau de la convention sociale qui affecte tous les instruments d'échange et les rend efficaces. Au surplus, les vertus que l'on prête à l'or ne sont pas complètement indélébiles. Les pièces anglaises de ce métal avaient subi, peu avant 1773, un notable affaiblissement du poids, par frottement, sinon par rognage, sans que la valeur faciale ait été changée. Dans cette situation, il ne pouvait plus être l'arbitre infaillible que l'on suppose'.

En ordonnnant la refonte des monnaies devenues légères et la frappe de guinées, de leur multiples et de leurs sous-multiples, remises aux normes, on dira sans doute et non sans justesse que, de cette manière, le cabinet de Saint-James avait rendu son autorité à l'or. Laissons un instant l'occurence de côté. Dans beaucoup de pays et, en Angleterre même, au $\mathrm{XVI}^{\mathrm{e}}$ siècle, les monnaies ont été affectées par des manipulations diverses : sur le poids, le titre ou aloi, la valeur nominale dans la circulation; bref, des dévaluations en tous genres. Elles déterminaient généralement une élévation des prix. Lorsque celle-ci, qui s'exprimait en monnaie de compte, n'excédait pas en sens inverse la diminution en teneur métallique des pièces de monnaie réelles, l'équilibre et le rôle diacritique de l'or, l'argent ou le bronze ne se trouvaient pas compromis. C'est la relation défendue par Malestroit dans sa célèbre controverse avec Jean Bodin. Mais le cas n'était pas universel. Souvent, obéissant à leur logique propre, les prix s'élevaient sans commune mesure avec la dévaluation monétaire et, d'ailleurs, au demeurant, et ce sans l'intervention d'une dévaluation aucune, par dessus la valeur commune ordinaire. A ce point de l'exposé, nous heurtons une représentation tenace du mouvement des prix. Celle qui le place dans la dépendance de la masse monétaire. En d'autres termes, la théorie quantitativiste, qui a été illustrée précisément et notamment par Jean Bodin, après quelques auteurs espagnols, et avant d'innombrables économistes et historiens.

Jean Bodin, on le sait, a mis en relation « l'enchérissement de toutes choses » avec l'arrivée des métaux précieux d'Amérique et l'augmentation consécutive de

\footnotetext{
1 La refonte fut décidée le 31 juillet 1773.
} 
la masse monétaire circulante. Il ne réduit pas cependant la hausse réelle à cette seule cause. Il en admet d'autres à côté sans s'appesantir et sans entreprendre de pondérer l'ensemble. Or, l'une d'elles venait de sévir et, ce, juste au moment où Malestroit écrivit ses Paradoxes : la très grave crise frumentaire de 1566, qui avait porté les prix des blés à un niveau extraordinaire avec séquelles sur le marché dans les années suivantes. Le phénomène serait anecdotique et de retentissement strictement limité, s'il n'était pas déjà survenu fréquemment auparavant, y compris dans les débuts du XVI ${ }^{\mathrm{e}}$ siècle, et s'il ne s'était pas reproduit par la suite et jusqu'à la fin. C'est là le principe de la fameuse révolution des prix qui a fait couler tant d'encre, que l'on peut vérifier dans les chroniques de l'époque. Le caractère de leader de la hausse assumé par les céréales n'est pas contestable et la publication de Hamilton ne change rien à l'affaire pour diverses raisons qu'il serait trop long d'exposer. L'effet concomitant de cette inflation d'un autre type a été que la monnaie métallique, les métaux précieux eux-mêmes, ont perdu de leur valence, de leur pouvoir libératoire dans l'échange avec des marchandises. Suivant le calcul que j'ai produit naguère « un kilo d'argent a eu pour équivalence en blé en Espagne [...] 45 hectolitres au début du XVI ${ }^{\mathrm{e}}$ siècle, 9 au début du XVII ${ }^{\mathrm{e}}, 15 \mathrm{au}$ début du XVIII et 6 à la veille du XIX ${ }^{\mathrm{e}}$ ». Car le mécanisme a continué de jouer, y compris en Angleterre après 1774, avec ses alternances de résipiscence et d'exacerbation. Etrange impavidité de la mesure de la valeur... ${ }^{2}$

On assiste donc à un renversement de sens remarquable. Ce n'est plus la monnaie réelle, intrinsèque, qui assigne la valeur aux produits, mais c'est la production qui détermine la valeur de cette monnaie, la hauteur de son acceptation sur le marché. Le passe-passe peut échapper à un observateur désincarné, uniquement attentif aux statistiques parce que les prix sont toujours exprimés en monnaie mais, cette fois, en monnaie de compte, en unités conventionnelles, à moitié fictives, qui lui cachent la dépréciation effective dans les échanges des pièces concrètes. Le rôle d'arbitre de l'or, de l'argent, du cuivre, etc., est fonction du contexte et ne s'exerce, à des niveaux variés, que dans une situation apaisée. Dans la même perspective, il leur arrive de récupérer partie de leur valence quand la production traverse une séquence heureuse. Ce que l'on constate à plusieurs reprises d'un siècle à un autre et, plus spécialement au XIX', à la fin duquel la surabondance en Europe, aggravée par les importations de blé américain, a entraîné une sévère dépression des prix. L'exemple des céréales a été retenu parce que le plus voyant, le plus probant. Il va de soi qu'une analyse fouillée et aussi exhaustive que possible serait nécessaire pour affermir la conclusion. Mais il serait hors de propos, ici, d'entrer dans toute la complexité voulue, qui oblige, entre autres travaux, à l'autopsie et à la réécriture de la relation d'Irving Fisher, avec la distinction entre les circuits : lévogyre et dextrogyre ${ }^{3}$.

Objectera-t-on que les raisonnements ci-dessus ne sont pas sortis d'une économie à base de monnaie métallique ? Cela manquerait de consistance. L'expé-

2 Hamilton (1934). Discuté dans Morineau (1985). La citation est à la page 596.

3 Pour l'explication de ces termes et les équations afférentes, se reporter à Morineau (1985) et, tout récemment, à l'article Quodlibet (2001). 
rience du $\mathrm{XX}^{\mathrm{e}}$ siècle, avec sa cascade de dévaluations, ses décrochements de l'étalon-or, son ajustement perpétuel aux conjonctures aussi bien de marché que de politique ramènerait promptement à un bon sens rassis. Les cours des matières premières et des denrées tropicales : coton, café, cacao, arachides, letchis, vanille, etc., oscillent au gré du volume des récoltes, à moins d'un accident extérieur susceptible d'exciter la demande. La monnaie de compte persiste à libeller l'étiquette des prix mais elle a perdu presque intégralement un rattachement à une monnaie réelle, sauf symboliquement et encore ! A laquelle se raccrocher au demeurant? Il y a vingt ou vingt-cinq ans, les trois monnaies des Pays-Bas, de l'Allemagne et de la Suisse se trouvaient à peu près à parité entre elles à 2,25 francs français contre une unité. D'après le dernier cours du change que j'ai consulté, le florin, en 2001, en vaut 2,9766 ; le deutschemark : 3,38538; le franc suisse : 4,44015. Et j'en reste à des coupures, certes fiduciaires, mais palpables. Que dire des virements bancaires, des transactions boursières, du yo-yo des actions ? Lorsque certaines de ces dernières s'élèvent à des sommets dans la cotation puis s'effondrent, comment faut-il comprendre ces variations ? De quelle monnaie a été constituée la fortune des porteurs ? Qu'ont-ils perdu avec la chute ? Dans les deux cas, une monnaie qui n'existait pas ? L'assurance d'une opération blanche ne les rassérénerait point. Plutôt que de s'avancer dans les arcanes d'une tentative d'explicitation, on se contentera de ce constat : le premier des avantages proclamés de la monnaie est tout à fait friable, n'a été que passagèrement évident et l'on s'interrogera avec circonspection sur la légitimité de disserter à son propos comme s'il s'était agi de son aube à notre temps et en dépit du nom maintenu, de la même chose.

\section{INSTRUMENT D'ÉCHANGE}

Ce caractère est, pour une partie, solidaire du précédent. C'est celui-ci qui la fondait en existence, d'après certains auteurs, et qui permettait de la séparer sans appel de la paléomonnaie. Georges Depeyrot a écrit : " Pour avoir une fonction monétaire complète, du moins telle que définie par Aristote, il fallait que l'objet en question puisse être échangé contre plusieurs marchandises différentes ». Ce qui n'était pas le cas, selon lui, des objets précédents : barres de sel, colliers wampum, bracelets de bronze, haches, etc., mais l'apanage de la vraie monnaie, qui renvoie à l'immutabilité et à la pérennité de sa valeur. En accord avec son prospect d'ensemble, il ajoute en un autre endroit : " Le phénomène monétaire n'existe et ne peut exister que dans un cadre de frappes importantes. La conception du caractère marginal du numéraire ne me semble en aucun cas correspondre à une réalité historique ». L'affirmation est sans doute discutable mais elle éveille opportunément l'attention sur une autre circonstance et condition de l'exercice de la monnaie en tant qu'instrument d'échange. Je veux parler de ce que l'on appelle la monétarisation de l'économie, qui, en fait et avec tout autant de pertinence, pourrait être qualifiée de monétarisation de la société. C'est la diffusion dans le public des pièces de monnaie réelles et leur utilisation dans les transactions marchandes ${ }^{4}$.

\footnotetext{
${ }^{4}$ Depeyrot (1995), p. 33 et p. 5.
} 
La monétarisation est le serpent de mer de l'histoire. Plus ou moins brusquement, à un moment du développement de son récit, l'historien introduit le concept de la monétarisation, déclare que le pays dont il s'occupe, à l'époque qu'il aborde, est entré dans le système du marché alimenté, sinon gouverné, par la monnaie, la monnaie réelle, des pièces. Ordinairement, il y a un fait déclenchant à l'origine : l'organisation de l'empire athénien au $\mathrm{V}^{\mathrm{e}}$ siècle avant Jésus-Christ ; la dispersion des trésors du Grand Roi de Perse par Alexandre ; le transfert à Rome par OctaveAuguste des richesses de l'Egypte ; la découverte de mines de métaux précieux et, on s'en doute, de manière suréminente, de celles de l'Amérique au $\mathrm{XVI}^{\mathrm{e}}$ siècle. Superficiellement, on se demanderait pourquoi le processus a dû se répéter par saccades, si l'on ne prenait pas en compte des retours à une économie de troc ou de nature, périodiques eux aussi : appauvrissement des Guerres du Péloponnèse ; crise du système monétaire de l'empire romain au $\mathrm{III}^{\mathrm{e}}$ siècle après Jésus-Christ ; stérilisation ou/et pillage des trésors ; fuite des espèces vers l'Orient; disette et faim du numéraire, de l'or, surtout, au $\mathrm{XV}^{\mathrm{e}}$ siècle. L'ennui a longtemps résidé dans l'absence de précisions chiffrées, voire de documents suffisamment loquaces pour justifier les climats des marchés. Cette pénurie a été palliée depuis une trentaine d'années par les remarquables progrès des numismates qui sont parvenus à mesurer de manière plausible le volume des émissions monétaires d'un passé, y compris de plusieurs très reculés. L'avancée n'est pas, cependant, complètement décisive. Il faudrait pouvoir entrer dans les canaux de la distribution, atteindre les utilisateurs potentiels, peser l'importance de la monnaie dans leur pratique, apprécier la régularité .

Quelques notations concrètes pour des pays, considérés en certains siècles, comme déjà monétarisés invitent à la prudence. Rappelons que Hamilton, luimême, avoua que les quantités de métaux précieux débarqués en Espagne au $\mathrm{XVI}^{\mathrm{e}}$ siècle (et, en principe ou en théorie destinés à la frappe) étaient presque dérisoires en face de la masse des monnaies qui aurait été nécessaire pour acquitter la totalité des récoltes si elles avaient été mises sur le marché. Quand, en fonction du «stock » monétaire de la France au tournant des XVII ${ }^{\mathrm{e}}$ et XVIII ${ }^{\mathrm{e}}$ siècles, on sonde de quel quotient chacun des sujets aurait pu disposer, on découvre le chiffre mince de 25 livres tournois. En prenant pour diviseur le nombre des chefs de famille (supposée forte de 4 personnes), on parviendrait à la somme plus roborative de 100 livres tournois, insuffisante néanmoins pour satisfaire aux besoins financiers des achats au marché. En outre, cette arithmétique sommaire est désinformante. Elle égalise indûment la richesse dans la société française. Mais les manouvriers et artisans, au nombre de 2 millions selon Vauban, gagnaient au mieux, quotidiennement, 10 sols au jour le jour, qu'il leur fallait presque impérativement dépenser immédiatement. D'où une limitation du numéraire mis en circulation, sans parler de la qualité des pièces. De manière générale, l'autosubsistance était un idéal très recherché, même si l'on a eu tendance à en exagérer la domination. Et il y avait force « astuces » pour restreindre les débours en argent : des règlements en nature, des comptabilités à moitié, jusqu'aux trocs tacites ou déclarés. La monétarisation ne régna pas véritablement, la monnaie sonnante et trébuchante

5 Commode mise au point des travaux des numismates dans le compendium très bien fait de Cécile Morrisson (1992). 
ne devint qu'au XIX ${ }^{\mathrm{e}}$ siècle un instrument d'échange ubiquiste, avec le développement du salariat et le paiement en pièces. La remarque vaut pour la plupart des autres pays ${ }^{6}$.

Pareillement y a-t-il lieu d'en rabattre sur les « hémorragies » prétendues du numéraire à destination des horizons du commerce lointains. L'Extrême-Orient, surtout, a chatouillé les imaginations. Peut-être les 100 millions de sesterces que Pline l'Ancien voyait s'évaporer chaque année vers l'Inde ont-ils été pour quelque chose dans la mauvaise réputation? L'assertion et le chiffre sont incontrôlables. Les trouvailles de monnaies romaines dans le sud de l'Inde et en Cochinchine témoignent de l'existence d'échanges mais ne démontrent rien quant aux quantités exportées et il est peu probable que les marchandises énumérées à côté de l'or, une cinquantaine d'années auparavant, par Strabon, aient disparu des cargaisons. Lorsque l'on se transporte au XVI $\mathrm{XV}^{\mathrm{e}}$ siècle et à l'exploitation directe par les Portugais, on est frappé de la modicité de certains envois préjugés dispendieux : par exemple, les 50.000 piastres qui devaient servir à l'acquisition du poivre, à une époque où il en arrivait d'Amérique, chaque année, plus d'une dizaine de millions. Les Hollandais reprirent d'une certaine manière les us de leurs prédécesseurs. Ils tirèrent parti des ressources de l'Asie elle-même, s'instituant les intermédiaires et les transbordeurs entre les différentes contrées et se procurant les denrées de l'une avec les produits de l'autre. Ils réduisirent ainsi leurs besoins en argent frais en provenance de la métropole : de manière assurément drastique au $\mathrm{XVII}^{\mathrm{e}}$ siècle. Moins bien implantés, leurs rivaux ne pouvaient que les envier et s'en remettre, eux, aux vertus du numéraire. Le parti pris a cependant été mal compris. On l'a assimilé à un déversement incessant, intarissable, involontaire, pernicieux et sans recours. Dans la réalité, la sortie d'argent était seulement l'amorce d'une transaction et la promesse, sinon la garantie, d'un profit consécutif. Thomas Mun l'a expliqué. En aucun cas, au surplus, l'écoulement à l'extérieur n’assécha radicalement la nappe des espèces présente dans les pays européens. « Tombeau de l'argent » est une belle formule appliquée à l'Empire du Grand Mogol : aussi exagérée que controversable. En outre, inlassablement, les concurrents des Hollandais cherchèrent à les imiter. Ils n'y réussirent pas durablement du premier coup. Les Anglais y parvinrent cependant après avoir mis la main sur le Bengale. Ils réglèrent avec de l'opium les énormes chargements de thé qu'ils achetaient à Canton ${ }^{7}$.

Taxer d'archaïsme les pratiques décrites ci-dessus, au nom de la modernité alléguée d'une économie monétarisée, serait plutôt léger eu égard à la carrure et à l'envergure des partenaires intéressés. Fréquemment flétri du sobriquet infamant de troc, l'échange marchandises contre marchandises régissait, au fond, de grands pans du commerce en Europe même. Lorsque les Hollandais allaient chercher du sel, de l'eau-de-vie et du vin en France, ils ne s'acquittaient pas avec des pièces de monnaie mais avec ce qui leur revenait de leurs ventes de draperies et d'épices.

6 Calculs rapides effectués à partir de Spooner (1972).

7 Par suite d'un changement dans la structure des échanges en Extrême-Orient, les Hollandais durent augmenter les quantités d'argent qu'ils exportaient en Asie. 
Les Anglais étaient des plus attentifs à ne remporter de la Rochelle et de Bordeaux que l'équivalent de leurs propres réalisations et ils furent suivis dans ce système, au XVIII ${ }^{\mathrm{e}}$ siècle, par les Suédois et par les Russes. On a souligné l'emploi assez abondant par les Hollandais des rijks - et des leeuwendalers dans l'espace balte. Attman a même tenté d'en calculer le montant. Mais de là à resserrer les échanges à ce seul aspect, la marge est importante et l'étrangler serait oublier les harengs, le poivre et les petites étoffes, qui, ensemble, constituaient probablement la part prépondérante à Gdansk et à Koenigsberg. Autre secteur supposé gros mangeur de devises : la Méditerranée orientale ou Levant. Au XVII ${ }^{\mathrm{e}}$ siècle, les Hollandais marchèrent sur les brisées des Anglais qui, eux-mêmes, s'étaient inspirés des Vénitiens du $\mathrm{XV}^{\mathrm{e}}$ siècle, pour y trafiquer de leurs draps. Ils purent ainsi se passer de l'envoi de pièces d'argent et, suprême exploit, se rendre capables d'offrir leur crédit à Smyrne et Istamboul à des concurrents impécunieux. Comme en ExtrêmeOrient, le négoce avec du numéraire était un pis-aller, juste bon pour les marchands dépourvus d'une contrepartie en nature. Les Marseillais furent dans cette situation jusqu'au début du XVIII ${ }^{\mathrm{e}}$ siècle. La vogue des londres et des londrins languedociens leur permirent de faire pencher la balance de l'autre côté. Instrument d'échange, la monnaie l'a bien été. Non le seul. Sa prééminence est discutable. Souvent, elle n'est intervenue qu'à titre de moyen supplémentaire ou complémentaire, voire d'appoint. Ou de marchandise : prisée par ceux qui, pour diverses raisons, la recherchaient, profitable pour ceux qui la décaissaient ${ }^{8}$.

Le mercantilisme, voué aux gémonies (lui ou sa caricature) ne se glisse pas toujours dans ces transactions croisées et décroisées. Quand cela eût été, l'opprobre aurait-il été fondé ? Mais le reproche conjoint d'archaïsme, qui nous a paru quelque peu déplacé, ne peut-il pas être retourné ? La monnaie, considérée, à son tour, dans sa version métallique, comme un outil de paiement obsolète ou en voie d'obsolescence, déjà, au XVIII' ${ }^{\mathrm{e}}$ ou au XVII ${ }^{\mathrm{e}}$ siècle ? Elle était peu à peu supplantée dans le grand négoce par la lettre de change ; qui, par la grâce des endossements, en épargnait quatre, cinq ou six fois le débours. Et combien plus à la Banque de Change ou Wiselbank d'Amsterdam par le jeu des virements de partie à partie, fonctionnant à partir d'un dépôt restreint, mobile ; constamment sollicité et constamment réalimenté. On sait que le solde global de l'établissement qui perdurait d'exercice en exercice, sans être trop grossi ou excessivement renouvelé, n'a égalé qu'exceptionnellement l'arrivage d'une seule flotte américaine en Andalousie, pour ne rien dire du cumul éventuel d'une année sur l'autre. Sans doute, pourra-t-on crier à l'abus de langage et retrouver la monnaie, derechef, sous des défroques. Le raisonnement n'est pas apparemment dépourvu de plausibilité puisqu'en définitive chaque opération était assortie d'un pouvoir libératoire et qu'au terme la monnaie métallique récupérait son magistère. Cependant, le rapport est ambigu. Tant qu'elle était en circulation, la lettre de change était susceptible de perdre son honorabilité - au sens propre et financier. De facto de cesser d'être monnaie, même fiduciairement. Les deux instruments ne sont donc pas

8 C'est l'effigie de l'Impératrice qui assura le succès sur les piastres espagnoles des Maria-Theresien Thalers au Levant dans la seconde moitié du XVIII ${ }^{\mathrm{e}}$ siècle, en dépit de la (très) légère infériorité en valeur intrinsèque. Les Marseillais en firent un grand commerce qui compensa partiellement la désaffection qui avait commencé de toucher le débit des draps. Les Maria-Theresien Thalers coururent au Proche-Orient et en Afrique orientale jusqu'au XXe siècle. $C f$. Courdurié et Rebuffat (1960). 
exactement superposables. Essentiellement, ils ne sont pas identiques. La dénomination commune à laquelle on arriverait à se raccrocher pour conjurer leur dissociation s'avère un genre d'élucubration et de leurre intellectuel. Or, la lettre de change et les autres substituts para - ou pseudomonétaires, plus ou moins dérivés, ont eu le vent en poupe et l'on est passé progressivement d'un type de monnaie à un autre. Ou, en termes réalistes, il n'y a pas eu transmission du même au même. Après avoir été déchue de sa position d'instrument d'échange unique, la monnaie métallique, phare et champion de la «monétarisation », se voit dépossédée de son caractère de « modernité » absolue. L'archaïsme des échanges, de son côté, ne se porterait pas si mal avec les accords de compensation signés de nos jours entre deux nations pour harmoniser leurs économies par la réciprocité.

On ampute gravement l'étude de la monnaie quand on la confine dans son rôle d'instrument d'échange. Aristote sert de caution à l'attribution dans son Ethique de Nicomaque : «Toutes les choses faisant objet de transaction doivent être, d'une façon quelconque, commensurables entre elles. C'est à cette fin que la monnaie a été introduite, devenant une sorte de moyen terme, car elle mesure toutes choses ». On oublie de prolonger la lecture. Le paragraphe se termine pourtant de la manière suivante : «Elle (la monnaie) porte le nom de nomisma (en grec) parce qu'elle n'est pas un produit de la nature mais de la loi (nomos, en grec) ». Or, la formule mérite de retenir l'attention. Elle estampille un caractère de la monnaie qui, sous un certain angle, a probablement été le premier et qui, quoi qu'il en soit, l'a toujours accompagnée. Autant qu'une « réalité sociale » dans le langage de Simiand, elle a été et elle continue d'être une « réalité politique ». Ou pour mieux dire, elle a été, elle est réalité politique et réalité sociale, simultanément et l'une par l'autre. C'est l'empreinte de l'autorité posée sur un morceau de métal qui distingue la pièce du lingot taillé et certifie en principe sa valeur dans la circulation. Remontant aux origines, à Crésus et à Darius, Georges Le Rider a signalé les interventions des souverains soit pour obtenir l'homogénéisation du versement des impôts, soit pour employer la monnaie en cadeaux et en récompenses, en dépenses de prestige et en subventions aux alliés, en primes de recrutement et en rétributions de mercenaires. La dépendance a été renforcée avec l'assignation d'une zone de validité, avec le prélèvement d'un seigneuriage et, last but not least, avec les manipulations sur le poids, sur le titre et sur la valeur nominale. Ces pratiques, se perpétuant de siècle en siècle, ont émaillé toute l'histoire de la monnaie, l'ont façonnée autant et davantage que l'utilisation comme instrument d'échange?

Fréquemment, le pouvoir se manifestait avant même le début de la fabrication en levant un impôt sur les mines de métal. Battre monnaie, ensuite, était un privilège régalien, nonobstant délégations et fragmentations. Relevaient aussi de l'autorité la définition des caractéristiques des pièces : type, poids, aloi, valeur officielle dans la circulation, la détermination du taux du seigneuriage, le contrôle de la qualité des frappes, la punition des infractions jusqu'à la plus sévère à l'encontre du faux-monnayage, ipso facto assimilé au crime de lèse-majesté. Les souverains intervenaient encore sur le volume de la monnaie circulant dans leurs

\footnotetext{
9 Le Rider (2001).
} 
Etats. De manière directe, lorsqu'ils s'érigeaient en pourvoyeurs des ateliers, achetaient les matières premières, offraient des primes aux financiers qui les leur rabattaient, en décidant des refrappes, des conversions et des refontes. De manière indirecte en offrant aux détenteurs des prix alléchants du marc d'or et du marc d'argent pour qu'ils les portent aux Hôtels des Monnaies ; en provoquant la monétarisation forcée de leurs peuples par l'obligation promulguée de se servir de numéraire pour certaines transactions et, surtout, pour le paiement des impôts (les Ptolémées en Egypte et le Grand Mogol Akbar en Inde sont restés célèbres pour ce dernier chef). Enfin, les princes sont intervenus dans le volume des pièces en circulation et dans la dissémination par leurs dépenses somptuaires, diplomatiques, voire par des rançons à payer (cf. les rois de France Jean le Bon et François $\mathrm{I}^{\mathrm{er}}$ ), par les prélèvements ordinaires et extraordinaires (en temps de guerre) sur leurs sujets, pouvant entraîner la disette latente des disponibilités, la mauvaise rentrée des impôts, la fatigue et jusqu'à la révolte. Impossible à la vue de ce catalogue de nier l'emprise de l'Etat sur la monnaie.

Pour les personnes convaincues de la monnaie instrument d'échange et instrument d'échange exclusivement, les labyrinthes parcourus ne seraient que péripéties. A leur issue, elle recouvrerait sa fonction essentielle, au demeurant potentiellement jamais égarée. On peut, bien entendu, procéder en pensée à un tel télescopage. La monnaie est alors rendue à une condition idéale, exempte de toute éclaboussure et de toute compromission. Mais le renoncement afférent est-il légitime ? La monnaie est une « réalité » et, dans son existence, elle n'a pas à être extirpée de son environnement, de ses avanies et de ses avatars, de son maniement. La saine appréciation commande de tout retenir de ce qui l'entoure pour discerner son rôle et, donc, de ne pas la soumettre a priori à une « phantasmisation », complètement à rebours des situations concrètes. A commencer par ses efflorescences ponctuelles, hachées, répétées et par les conditions d'apparition et de disparition des bouffées. Je m'en suis tenu à des exemples appartenant à l'époque de la monnaie métallique. La prégnance de l'ingérence de l'Etat n'a fait que s'accentuer par delà. Je ne reviendrai pas sur sa position discrétionnaire quant aux cours et, à peu de chose près, quant aux émissions. Ses prélèvements et sa redistribution des ressources pèsent dans les deux sens sur la circulation et sur le pouvoir d'achat des particuliers. Il s'immisce dans la vie économique du pays à un degré qui n'avait jamais été atteint. Réagissant selon les circonstances en serrant ou desserrant le robinet du crédit, en relevant le salaire minimum d'insertion communautaire, en fixant arbitrairement le prix des produits qui renflouent ses finances : allumettes, tabac, essence à la pompe, etc. La monnaie est sans doute, toujours, nominalement l'instrument comptable de la mesure de la valeur, de l'échange. Elle a été dépouillée à l'unité de son efficience ${ }^{10}$.

${ }^{10}$ Je n'aborde pas ici le problème de l'efficacité sur le marché de l'argent considéré dans sa masse, qui entraînerait un développement hors de saison. Il impliquerait la relecture de la relation d'Irving Fisher et, en particulier, la reconnaissance des deux circuits : lévogyre et dextrogyre. Je renvoie aux travaux signalés dans les notes 2 et 3 . 


\section{RÉSERVE DE VALEUR, PRINCIPE DE DÉVELOPPEMENT ET RECENSION}

Lorsqu'il a effleuré la question de la conservation de valeur de la monnaie (habituellement désignée de « réserve »), Aristote avait visé simplement une utilisation différée. Dans un temps court, l'allégation n'était pas insoutenable, sauf coïncidence malheureuse avec le passage impromptu de turbulences sur les mercuriales. A long terme, la perspective est plus gandilleuse. Le Stagirite reconnaissait la possibilité d'une dépréciation de la monnaie mais s'en sortait en lui accordant un label de stabilité (ou de plus grande stabilité). Il n'envisageait ni l'envolée des cours pour cause de disette, avec, symétriquement, une baisse du pouvoir d'achat, ni les altérations et les décris cependant « légaux », phénomène décrit par Aristophane. Il est vrai que le parti à prendre par les possesseurs tournait quelquefois au dilemme : enfouir des pièces lourdes en spéculant sur le maintien de la valeur intrinsèque supérieure ou profiter immédiatement de la plus value nominale sur le marché ? Dans le cas d'une empirance, il n'y avait guère à tergiverser, sauf, par désespoir, à hésiter à jeter à la rivière les piécettes d'une très menue monnaie, devenue encore plus fluette, jusqu'à l'inconsistance (on en a des cas en France au début du $X^{\mathrm{e}}$ siècle). La conservation de la valeur ne se vérifiait que dans des conjonctures définies qui ne se sont pas toujours réalisées. Les déboires de l'aureus, de l'antoninianus et du denier à Rome sont connues et la volatilité persistante du prix du blé ${ }^{11}$.

La monnaie a-t-elle été l'unique concentré de la valeur et l'unique gardien ? A moins d'avoir l'amour d'Harpagon pour sa cassette, on n'en croira rien. La diffluence des trésors d'Egypte ramenés par Octave-Auguste vers la terre, dont le prix augmenta considérablement, trahit à la fois un relatif dédain et une préférence pour une richesse plus solide. Les deux réactions sont compréhensibles. L'adage nummus non facit nummos s'appliquait dans le premier cas ; le rendement escomptable de la propriété foncière (et des esclaves, autre levier des exploitations) dans le second. La terre a longtemps gardé, d'ailleurs, son pouvoir d'attraction. En France, jusqu'au troisième quart du XIX ${ }^{\mathrm{e}}$ siècle, au moins. Si, depuis ce temps, il a notablement régressé en général, au grand dam des paysans, il est des spéculations qui en ont gardé quelque chose et même qui en ont gagné : témoins les grandes fortunes qui se sont investies dans l'achat des vignobles prestigieux du Bordelais. La pierre, l'immobilier ont également tenté, voire des hobbies susceptibles de maintenir leur valeur, sinon d'acquérir quelque jour un lucre intéressant comme les tableaux, des collections variées, etc. De lui-même, l'argent est stérile. Il ne rapporte que bien employé, transformé, digitalisé. D'où le transfert non seulement dans des activités industrielles, commerciales, etc., mais encore, dans des actions et des obligations, genre SICAV et autres : nouveau « petit matelas ». L'or

${ }^{11}$ Les dévaluations ont foisonné d'un siècle à un autre. Les plus célèbres sont peut-être la manipulation monétaire au III $^{\mathrm{e}}$ siècle dans l'Empire romain, celle du roi Henry VIII en Angleterre au milieu du XVI ${ }^{\mathrm{e}}$ siècle, le lancement de la monnaie de vellon en Espagne sous les Philippe III et IV, le Kipperund Wipperzeit en Allemagne vers 1621-1622, la fin du règne de Louis XIV en France en attendant «l'expérience de Law » et les assignats. 
n'est plus une valeur refuge : c'est une des dernières vérités de La Palisse. Il avait itérativement survécu en tant que tel dans les coffres des Banques d'Etat par attachement désuet à une « relique barbare » (dixit Keynes). Il a largué les amarres parallèlement au naufrage des «bonnes » monnaies, de l'étalon métallique. Preuve, s'il en fallait encore une, que nous avons quitté un système pour un autre, que la monnaie n'est plus ce qu'elle a été ${ }^{12}$.

Quelle force majeure n'a-t-on pas attribué, pourtant, à l'or et à l'argent dans et pour le passé ! Diffuse, d'abord, insinuée et insinuante, finalement irrésistible. L'intrusion de la monnaie aurait lubrifié l'économie, accéléré le rythme des échanges, répandu la richesse et la prospérité : bienfaits merveilleux et miraculeux de la «monétarisation » dont nous avons constaté et le succès historiographique et les doutes qui en entourent la manifestation effective, l'extension en largeur comme en profondeur. On ignore délibérément les autres causes d'émoustillement des marchés. Par exemple, la mise en demeure des habitants d'un pays quelconque de se procurer un répondant monétaire pour satisfaire à l'exigence d'un impôt prélevé en espèces : lorsque l'empereur Auguste doubla le montant du tribut qu'il réclamait de la Gaule, il fallut bien que l'on s'en acquittât. Eventualité à répétition : la monétarisation a réagi bon gré mal gré à l'incitation fiscale et l'a suivie, restât-elle pelliculaire comme dans l'empire du Grand Mogol. Mais on a invoqué des effets de levier plus spécifiques. Il est vrai que le cas de l'Espagne au XVI siècle est très embarrassant car il en dément la dynamique. Qu'à cela ne tienne ! Récemment, des auteurs estimables et sincères ont relancé le postulat à propos de la France au XVIII siècle qui aurait été galvanisée par l'or du Brésil. Malheureusement, la partie proprement économique, sans préjudice de la partie statistique, souffre d'une espèce de conviction avant examen et, en outre, d'une carence catastrophique de l'information. Aurait-on eu plus de chance avec l'Angleterre, rivée au Portugal et à sa colonie par le traité de Methuen? La « révolution industrielle » serait-elle sortie toute armée ou presque des Minas Geraes ? Un coup d'œil sur les Customs Registers détruit l'illusion. De Londres et des out ports davantage d'or a été exporté qu'il n'en a été frappé à la Monnaie ${ }^{13}$.

Derrière ces affimations se profile un acte de foi invétéré depuis Karl Marx : la croyance dans l' « accumulation primitive » qui aurait été nécessaire pour rendre inéluctable le règne du capital. L'eût-elle été que la Hollande et la France en auraient pu bénéficier et réussir leur démarrage ou take off, plus ou moins en même temps que « la première nation industrielle ». La pensée du philosophe de Trêves n'était pas aussi simpliste, son schéma était articulé. C'était la dépossession de leurs moyens de subsistance, d'indépendance, qui avait livré les paysans et les artisans anglais, pieds et poings liés, à la classe des capitalistes et fait la for-

12 Dans les campagnes françaises, les draps de lit, qui s'empilaient dans les armoires, constituèrent bien une réserve de valeur pour les paysans et les paysannes jusqu'en 1940, sinon au-delà.

${ }^{13}$ Morrisson, C., Morrisson, Ch. et Barrandon, J.-N. (1999). Pour une analyse critique de cet ouvrage, voir le Quodlibet, cité à la note 3. Pour l'Angleterre, j'avais fait le dépouillement non encore publié des Customs Books conservés au Public Record Office. Les sorties d'or s'élevèrent entre 1703 et 1772 (inclus) à 53,4 millions de livres sterling environ. Durant la même période, les frappes, d'après sir John Craig (1953), à 30,7 millions, toujours environ, soit les deux cinquièmes. Où donc était passé l'or brésilien ? Comment peut-on lui attribuer par sa masse une influence indispensable et irrésistible? 
tune de ceux-ci. Les homologues français résisteraient, appuyés sur la propriété de leurs lopins, mais succomberaient à leur tour, un jour, fatalement. Je ne m'aventurerai pas dans un pilpoul sauvage autour des propositions de l'auteur du Capital. Mais j'apporterai une addition essentielle pour la description correcte de ce qui s'est passé. Le capital, réduit à lui-même, est impotent pour créer un courant de croissance. Il lui faut, on l'a déjà croisé, un agent de conversion adéquat. Son défaut explique les échecs de l'Espagne et du Portugal (dont je réserve le sondage approprié). Mais le multiplicateur d'efficience ne réside pas toujours ni uniquement dans une structure d'accueil économique, ni dans une invention technique. L'homme, dans certaines conditions, est, par son travail, un facteur de base. Les mises de fonds des compagnies anglaises pour l'expatriation des candidats à l'émigration en Amérique ont moins compté pour le développement des treize colonies que la force des bras déployée dans la conquête du sol, les essartages et les défrichements. Sans grosse infiltration renouvelée de monnaie, au demeurant. Mutatis mutandis, le grand essor des plantations antillaises au XVIII ${ }^{\mathrm{e}}$ siècle a reposé sur les sueurs et la fatigue des individus. Là, des esclaves ${ }^{14}$.

La fascination du capital a occulté ses ratages. Ils n'ont pas été rares, cependant, ni minces. Evoquons pour mémoire les initiatives de Colbert dans le commerce colonial qui furent à fonds perdus ; les « bulles » spéculatives en France et en Angleterre au début du XVIII ${ }^{\mathrm{e}}$ siècle : l'expérience de Law et la South Sea Company. Combien d'entreprises de moindre envergure se sont-elles effondrées après avoir vivoté, ou, même, un temps, remporté quelques succès. Les « révolutions industrielles » sont parsemées de cadavres économiques. Il a fallu que les Etats injectent de l'argent vite évaporé pour inciter des entreprises à s'implanter en certaines régions, soutiennent celles qui étaient au bord de la faillite sans, parfois, la leur éviter, essuient les aléas de la Bourse, du marché des changes, des krachs, etc. Ne commandant que très imparfaitement l'évolution de l'économie et les ruses, foucades, artifices osés ou frauduleux des spéculateurs habiles à jouer des mouvements monétaires bruts et sophistiqués. Ce dernier trait, qui n'est nullement inventé, parachèvera peut-être la démonstration, sauf pour ceux qui l'auront disqualifiée in petto comme une philippique exagérée et, par conséquent, insignifiante. Ces derniers se tromperaient sur l'esprit qui m'a animé. Je n'ai pas voulu me faire l'émule de Monsieur de Malestroit avec des « Paradoxes ». Et, comme tout un chacun, je partage l'opinion bien décidée d'une grande dame britannique : «I want my money in my pocket ». Mon propos a été autre. Comme je l'ai dit en introduction : appeler à une réflexion collective sur le sujet qui est à l'origine de notre rencontre, rendre un peu plus visible notre « princesse lointaine ». Que le résultat de la prospection aboutisse à une réévaluation sérieuse, sinon déconcertante et gênante, c'est probablement un fait. Mais s'il bouscule, ce qu'il bouscule est seulement une tradition, une façon de décrire sans décrire, muée en enseignement routinier, magistral et, pour comble, paresseuse.

${ }^{14}$ D'après les Customs Books, les sorties d'or et d'argent à destination des colonies anglaises en Amérique au XVIII ${ }^{\mathrm{e}}$ siècle furent infimes. L'esclavage aux Antilles françaises (et aux Mascareignes) a été d'une très grande importance pour la prospérité indubitable des ports métropolitains et la prospérité apparente du royaume tout entier. A Rome, sous le Haut Empire, le rendement d'un esclave pour son maître était d'un rapport assuré de 70 sesterces par an. 
Car la très grande majorité des commentateurs, des experts, des « professeurs d'économie » n'ont pas lu d'un bout à l'autre les traités d'Aristote : l'Ethique de Nicomaque et les Politiques. Ils en ont extrait, sélectionné, élu les phrases qui leur convenaient et qui leur ont paru positivement exprimer la vérité de la monnaie : mesure de la valeur, instrument d'échange, réserve de valeur. Ils n'en ont pas testé la validité. Avaient-ils même pris connaissance de l'intégralité des textes. Dans le premier ouvrage, le fondateur du Lycée partait d'un échange marchandises contre marchandises : " Prenons, par exemple, un architecte A, un cordonnier B, une maison $\mathrm{C}$, une chaussure $\mathrm{D}$. Il faut que l'architecte reçoive du cordonnier le travail de celui-ci et qu'il lui donne en échange le sien ». Il s'agit, en somme, d'un troc. « Toutefois, il est indispensable, auparavant, de les rendre égaux. Aussi fautil que toutes choses soient en quelque façon comparables, quand on veut les échanger. C'est pourquoi on a recours à la monnaie, qui est, pour ainsi dire, un intermédiaire ». L'Ethique de Nicomaque ne fournit aucune précision sur une pièce réelle. La monnaie en question apparaît de la sorte comme une simple unité de compte et l'on parlerait alors, non sans raison, d'un troc masqué sous la couverture monétaire. C'est dans les Politiques qu'Aristote est entré dans une description des caractéristiques matérielles : «La valeur fut d'abord définie par les dimensions et le poids, puis finalement par l'apposition d'une empreinte, pour éviter d'avoir sans cesse à les mesurer ». L'invention est liée au commerce à distance, «à la faculté de changer facilement de mains », à la référence implicite à la conservation de la valeur jusqu'à usage. Retour à l'Ethique pour l'étymologie de nomismata, la filiation avec nomos: la loi, la tendance à la stabilité ${ }^{15}$.

Telle quelle, la démonstration du Stagirite comporte des pétitions de principe, des achoppements du raisonnement, des incohérences et des aveux involontaires. L'échange, à l'origine, est créé par les besoins réciproques des partenaires. Pour satisfaire à la justice, préoccupation principale du philosophe, ils doivent être égalisés : ce à quoi pourvoit le recours à la monnaie. Mais, fidèle à la direction de son esprit, il suppose déjà, au préalable et par préalable, l'existence d'une égalité au niveau des besoins. Il n'envisage pas le cas d'un déséquilibre, d'une appétence de nourriture, pour prendre un autre de ses exemples, plus forte qu'un désir de chaussures. Déséquilibre qui se répercute dans le face à face monétaire, rompant l'équité parfaite dans la proportion et ruinant le mythe de l'immarcescibilité de la valeur de la monnaie. La conception d'ensemble explique la lacune. L'anecdote qu'il raconte à propos de Thalès aurait pu néanmoins lui mettre la puce à l'oreille. Vexé de s'entendre raillé à tout instant pour sa pauvreté, l'astronome mathématicien monta une spéculation fructueuse. Ayant prévu une récolte d'olives abondante, quand personne ne l'espérait, il prit à ferme à bon marché tous les pressoirs à huile de Milet et de Chios et les sous-loua le moment venu pour le prix qu'il voulut. Des opérations de monopole similaires étaient assez communes de la part des cités pressées par le besoin de se procurer des ressources. Elles relevaient de la chrématistique qu'Aristote vomissait comme une injure à la nature. Mais cette réaction morale d'un individu était impuissante à empêcher nombre de ses semblables à rechercher l'acquisition de richesses par le commerce et d'autres

\footnotetext{
15 Ethique de Nicomaque, V, 5 (1965). Politiques, I, 9, 1257a (1993).
} 
moyens litigieux. Isoler la monnaie des mouvements qui la brassaient et rebrassaient la privait de substance et, partant, comme vu plus haut, mutilait gravement la perception et l'analyse. Sans oublier que ce qui est sous la tutelle d'une loi et d'une convention peut être modifié, défait, dénaturé par une autre loi et une autre convention. L'insensibilité aux changements monétaires jure, pour qui veut bien faire le rapprochement, avec la richesse des gradations déployée à propos des constitutions ${ }^{16}$.

Soit en se trahissant, soit en laissant béants ses textes en certains endroits, Aristote, lui-même, libérait un large espace pour une poursuite de l'instruction. L'enquête à laquelle je viens de me livrer se trouve ainsi, de facto et de jure, justifiée. Sans cavillations ni contorsions. Elle n'est assimilable ni à un délit d'irrévérence, ni à un délit d'outrecuidance. Les formules du Stagirite conservent, bien entendu, leur pertinence propre mais celle-ci est dans la dépendance de la conception générale, éthique, normative par dilection et vocation, à la limite abstraite ou éthérée. Elles la perdent en grande partie dès lors qu'elles sont confrontées à la réalité qu'elles avaient niée. Elles sont impuissantes à rendre compte du phénomène de la monnaie dans la totalité de ses manifestations. Car les fonctions politiques et commerciales furent têtues comme l'illustreraient facilement, entre autres exemples, les manipulations de poids, de titre, de valeur et de substrat qui ont jalonné le passé de tous les pays et l'invasion triomphante de la vie économique par les échanges contre monnaie. L'influence d'Aristote n'a pas aidé, c'est le moins que l'on puisse dire, les penseurs et les écrivains qui se sont occupés du sujet. Les impedimenta ne venaient pas seulement de l'adoption, cependant persévérante, des définitions et des axiomes énoncés sur les équivalences, sur l'anathème de l'argent pour l'argent, etc. Ils tenaient encore et davantage à la manière de poser les problèmes. Invinciblement tirés vers une intellectualisation coupée par mimétisme, d'emblée ou très tôt, des réalités matérielles.

Il en résulta la persistance de l'élision de principe avec ses pétitions impératives ou optatives, incluses et implicites. Comme il fallait bien, nonobstant, se colleter avec les difficultés de chaque jour, et que l'on n'y était pas préparé, les opinions contradictoires proliférèrent : sur le bien-fondé des altérations de la monnaie, sur la proportion de l'or et de l'argent dans la circulation, sur l'interdiction ou l'autorisation de l'exportation du numéraire, sur la formation des prix, sur leur licéité et sur l'usure. Cela n'alla pas sans engendrer un certain babélien, dont les gouvernements et les « agents de l'économie » n'eurent pas cure, ordinairement, par-delà les précautions oratoires. Finalement, la réputation de la monnaie toucha le fond quand on la déclara «neutre » et que, en conséquence, on lui refusa une quelconque importance ${ }^{17}$.

16 Politiques, I, 11, 1258b.

17 Une liste des auteurs qui se sont occupés de la monnaie après Aristote et jusqu'au début du $\mathrm{XIX}^{\mathrm{e}}$ siècle est trop fournie pour être reproduite ici. Parmi ceux qui exercèrent une grande influence, on peut citer saint Thomas d'Aquin, Jean Bodin (qui avait été précédé en Espagne par Martin de Azpilcuesta), David Hume. L'épineuse question du prêt à intérêt et de l'usure donnera une bonne idée à la fois des rigidités conceptuelles et des acrobaties dialectiques déployées pour parvenir à une auto- 
L'imprégnation méthodologique explique partiellement sans doute l'indifférence habituelle des auteurs jusqu'au XIX ${ }^{\mathrm{e}}$ siècle pour les précisions chiffrées. L'absence de statistiques régulières de leur temps était une autre cause, bien que tous n'auraient eu droit à s'en prévaloir, à commencer par Condorcet, inspecteur des Monnaies ! L'impression de rhétorique ingénieusement ou laborieusement appliquée en sort accentuée. Rétrospectivement, la situation heuristique a été améliorée par un gros effort des historiens, soutenus par le récent et vigoureux coup de main des numismates. Nous possédons désormais des tableaux quoique inégalement riches du commerce et de la production agricole et industrielle, d'une dizaine de séries continues des frappes monétaires sans préjudice de framents épars, abondance de profils démographiques, surabondance de prix, surtout du blé. Leur interprétation n'a pas toujours décollé de la routine et des clichés qu'elle charriait. Il est même surprenant de constater une espèce d'incapacité à s'en arracher et à se rendre aux plus criantes évidences des documents et des graphiques exhibés : le ballet des opinions sentencieuses autour des prix en est la concrétisation. L'incursion dans la mathématique et, aujourd'hui, a fortiori, dans la cliométrie n'écarte pas les embûches et les déformations telles que la désertion du suivi chronologique, la réduction abusive à des moyennes de tout poil, le lissage vicieux des courbes en rapport, la ruée sur les corrélations immatures. Non condamnable en soi, le penchant actuel pour la macroéconomie, qui exigerait une recrudescence de rigueur, n'est pas moins exposé aux séductions perverses, aux dérives et aux impasses ${ }^{18}$.

L'appareil statistique s'est beaucoup étoffé et raffiné depuis les débuts du $\mathrm{XIX}^{\mathrm{e}}$ siècle. Il a cessé de balbutier pour offrir des matériaux fermes et fiables. Du moins, en principe : on ne peut ignorer les fantaisies patriotiques des pays en proie aux virus de la publicité et de la propagande, ni, chez les autres, quelques troubles et perturbations dans la fabrication et la présentation. Les économistes patentés affichent néanmoins la sûreté dans les convictions et, du moins par épisodes, la sérénité de Bouddha. Le cordon ombilical n'a pas été sectionné. En 1996, l'un d'eux, Willem Korthals Altes, inaugurait son livre, Van pond Hollands tot Nederlandse gulden. De geschiedenis van de Nederlandse geldeenheid, par le rappel des trois fonctions « aristotéliciennes », avec le commentaire spontané : « Deze func-

risation «sans péché ». Dans quelle mesure ces dissertations eurent-elles un écho dans la pratique et des hommes d'affaires et des gouvernements, le problème n'a guère été étudié dans toute son étendue avec la volonté d'aboutir.

${ }^{18}$ Condorcet aurait eu facilement à sa disposition la récapitulation des frappes monétaires en France au XVIII ${ }^{e}$ siècle qui avait été remise au Contrôleur Général des Finances Joly de Fleury. C'est elle que j'ai publiée dans le recueil de John Day (1984). Pour la petite histoire, cet article intitulé : «Les Frappes monétaires françaises de 1726 à 1793. Premières considérations » n'a jamais été cité, $a$ fortiori pris en compte, ni probablement même regardé, par les collègues français avant le travail de Jean-Noël Barrandon, Cécile et Christian Morrisson qui sortit en 1999 (cf. note 13). Beau témoignage de conscience professionnelle et de considération pour l'œuvre d'autrui. Parmi les dix-huit ou vingt personnes concernées qui auraient normalement $d \hat{u}$ en faire état, en raison des sujets qu'elles traitaient, figure horresco referens le préfacier ( $\mathrm{sic}$ ) du Cahier Ernest Babelon numéro 7. Qui avait commis entre temps un Ancien Régime de Louis XIII à Louis XV (1610-1770) - eh ! oui ! -, partie d'une Histoire de France, et dont on ne vantera jamais assez le charme, la rigueur et l'honnête sous-information. Quinze ans seulement ! Je ne dirai rien aujourd'hui des autres abstinents. 
ties kunnen als uitgangspunt voor een behandeling van de monetaire geschiedenis dienen » (ces fonctions peuvent servir de point de départ pour le traitement de l'histoire monétaire). Il optait ainsi délibérément pour la continuité sans rupture du plus ancien au plus récent, pour l'identité profonde de nature et d'usage de l'unité monétaire : de la livre archaïque (pour les Pays-Bas) au florin de la reine Beatrix. Et le raisonnement, par similitude, vaudrait pour tous les autres étalons de valeur de par le monde. L'affirmation est cavalière. Ce qui a changé n'est pas seulement la forme (papier ou écriture contre métal), mais la masse en circulation, les emplois diversifiés, l'absence de garant immédiatement consultable et tangible, la banalisation synonyme d'incuriosité quant à l'intrinsèque, l'incertitude sur l'être, une perplexité allant jusqu'à l'inquiétude sur la consistance et la durabilité. Le commun peuple la reçoit quasi mécaniquement sous forme de salaires et de rentrées qu'il souhaite les moins aléatoires possibles. On sait qu'elle est entre les mains des gouvernements, des capitalistes et, en outre, des mystérieux opérateurs qui spéculent sur les variations du change, sur les cours montant et descendant des actions, sur les anticipations de résultats des entreprises avec l'épée de Damoclès d'un bouillon sinistre suspendue au-dessus de leurs têtes. Le rabâchage des caractéristiques aristotéliciennes serait tout à fait inhabile à éclairer leur lanterne. Les politiques monétaires, d'un pays à un autre, d'un temps à un autre, tanguent et roulent comme si l'on n'avait aucun repère fixe, aucun pôle de stabilité, aucune doctrine de conduite indéfectible. D'ailleurs, les experts se déchirent dans tous les compartiments de leur «science » et prônent les moyens de gestion les plus contradictoires, sans trop s'attarder ni revenir sur les conséquences. A la différence des augures de Cicéron, les Prix Nobel d'économie ne rient pas sarcastiquement en se regardant. Bref, c'est le pot-au-noir.

La délicatesse d'appréciation, mieux ou pire l'indécision, lèvent aussi bien dans la réalité qu'à la fin de cette enquête. Les études de détail n'en souffrent pas de contrecoup irrémédiable, à condition, toutefois, d'être correctement replacées dans leur niche d'intelligibilité, rattachées à un état bien délinéé de la monnaie. Celle-ci devait être appréhendée d'après l'invitation de ce colloque, en quelque sorte en pied, comme « personnage de l'histoire ». Il fallait, je crois, que cette annonce et ce projet, indispensablement, s'assortissent de «l'histoire du personnage ». Tant pis si elle nous exhérède un peu de notre confort métaphysique. «Want we weten nu wel wat geld doet, maar nog niet wat geld is ». Je conclus sur cette citation de notre confrère hollandais Menno Polak : «Parce que nous savons bien maintenant ce que fait l'argent [je nuancerai min of meer], ce que fait l'argent mais pas encore ce qu' est l'argent ${ }^{19}$.

\section{BIBLIOGRAPHIE}

Aristote, Ethique de Nicomaque, V, 5, trad. de Jean Voilquin, GF Flammarion, Paris, 1965.

Aristote, Politiques, I, trad. de Pierre Pellegrin, GF Flammarion, Paris, 1993.

Courdurié, Marcel, et Rebuffat, Ferréol : Marseille et le Négoce Monétaire international (1785-1790), Chambre de Commerce et d'Industrie de Marseille, 1960.

19 Polak (1999), p. 4. Auteur en outre des deux volumes intitulés : Historiographie en economie van de «muntchaos ». De muntproductie van de Republiek (1606-1795) (1998). 
Craig, John, The Mint, Cambridge University Press, 1953.

Day, John, Etudes d' Histoire Monétaire XII ${ }^{e}$-XIX ${ }^{e}$ siècle, Presses Universitaires de Lille, 1984.

Depeyrot, Georges, Histoire de la Monnaie des origines au XVIII sièle. I. Introduction. De l'Antiquité au XIII ${ }^{e}$ siècle, Moneta, Wetteren, 1995.

Hamilton, Earl J., American treasure and the the price revolution in Spain 1501-1660, Harvard University Press, Cambridge, Mass., 1934.

Le Rider, Georges, La naissance de la monnaie. Pratiques monétaires de l'Orient ancien, Paris, Presses Universitaires de France, 2001, Coll. L'Histoire.

Morineau, Michel, Incroyables gazettes et fabuleux métaux. Les retours des trésors américains dans les gazettes hollandaises (XVI ${ }^{e}$-XVIII ${ }^{e}$ siècles), Cambridge University Press et Maison des Siences de l'Homme, Paris, 1985.

Morineau, Michel, «Quodlibet. De l'or brésilien, de la macroéconomie et de la croissance économique au XVIII ${ }^{\mathrm{e}}$ siècle en France et en Angleterre », in Revue d'Histoire Moderne et Contemporaine, $2001, \mathrm{~N}^{\circ} 2$.

Morrisson, Cécile, La Numismatique, Paris, Presses Universitaires de France, 1992, Coll. Que Sais-je?

Morrisson, Cécile, Morrisson, Chistian, et Barrandon, Jean-Noël : Or du Brésil, Monnaie et Croissance en France au XVIII siècle, Paris, Editions du C.N.R.S., 1999, Cahiers Ernest-Babelon 7.

Polak, Menno S., Historiographie en economie van de «muntchaos». De muntproductie van de Republiek (1606-1795), 2 vol., Amsterdam, NEHA, 1998.

Polak, Menno S., Wat is monetaire geschiedenis ? (adresse prononcée au Congrès annuel de la Koninklijk Nederlandsch Historisch Genootschap), 1999.

Spooner, Frank C., The International Economy and Monetary Movements in France, 1493-1725, Harvard University Press, Cambridge, Mass., 1972.

\section{SYNOPSIS}

\section{LA SERVANTE INTRONISÉE MAÎTRESSE}

Les textes d'Aristote n'ont pas été lus avec suffisamment d'attention. La plupart des successeurs s'en sont tenus aux « trois fonctions ». Ils ont négligé le caractère politique, malgré la référence explicite au nomos. Ils ont amalgamé sans état d'âme monnaie réelle et monnaie de compte, en suivant la confusion implicite que leur suggérait le rapprochement de deux passages du philosophe. Ils ont, de la sorte, passé sous silence les variations de valeur intrinsèque et les manipulations monétaires, qui existèrent bien, cependant, dès les temps classiques de la Grèce et a fortiori à l'époque hellénistique. Ils n'ont pas réfléchi aux conditions spécifiques dans lesquelles s'était placé le Stagirite pour justifier l'espèce de neutralité de l'échange qu'il défendit. Ils ont tablé comme lui sur l'égalité entre les partenaires de leurs besoins réciproques. En conséquence et logiquement, ils ont ignoré les situations de déséquilibre existant sur les marchés et, en dépit de Thalès, les péripéties capricieuses des spéculations.

En aucun cas et d'aucune manière, la monnaie n'a été un ectoplasme et ne l'est, encore moins, aujourd'hui. Dès l'origine, elle a été considérée concrètement comme un bien et, à ce titre, est entrée normalement dans la circulation. Ses « trois fonctions » ne sont pas un mythe mais elles doivent être soupesées dans leur exercice. Sa mesure de la valeur a été plutôt épisodique dans le passé, à cause de la volatilité des prix des marchandises qu'elle était censée acquérir. Elle n'a pas été l'instrument unique des échanges, ni, parfois, le principal, contrairement à l'éter- 
nelle ritournelle sur les fuites de l'argent. Elle n'a pas été davantage le seul conservatoire de la valeur, longtemps concurrencée par de plus solides garants, dont la propriété foncière et, aujourd'hui, par divers autres. Par contre, son rôle politique en tous genres : pour le paiement des impôts, pour la levée d'emprunts, pour la solde des soldats, pour les dépenses de la guerre a été indûment occulté, quoiqu'il ait été souvent fondamental.

L'attirance des métaux précieux a conduit à exagérer leur rôle dans la vie économique et à leur prêter, par le truchement de la monnaie, un rôle démiurgique. En fait, ils doivent être replacés dans leur environnement, conjugués avec le travail des individus et pondérés dans leur effet contingent jusqu'au XIX ${ }^{\mathrm{e}}$ siècle. C'est alors que la monnaie est devenue de plus en plus présente en Europe occidentale avec la transformation de l'économie et de la société. La création des grandes fabriques ruineuses de l'artisanat individuel, l'exode des paysans sans terres vers les villes, l'accélération de la pratique du salariat dans la population, le développement d'un machinisme de plus en plus onéreux, la dépendance accrue des établissements de crédit, autant de leviers pour une mutation qui se continue sous nos yeux sans désemparer. La monnaie est devenue ubiquitaire avec la connotation d'impérialisme, son emploi contraignant aux niveaux les plus humbles et, en même temps, susceptible de grandes complaisances aux plus hauts, son action oscillant entre la prise de bénéfices, l'OPA et la banqueroute. Mais ce n'est plus la même servante élevée au rang de maîtresse, un jour couronnée, un jour marâtre. Princesse pour les uns, mégère pour les autres, et, toujours, nonobstant, à la merci d'un gouvernement impécunieux ou d'un rush d'opérateurs en Bourse. Il ne s'agit plus d'une métamorphose de ses outils, encore que celle-ci soit grosse d'informations nouvelles, mais d'un changement fondamental. La science économique serait bien avisée de revoir ses définitions. 